\title{
Casimir-cavity-induced conductance changes
}

\author{
Garret Moddel $\odot$, Ayendra Weerakkody, David Doroski $\odot$, and Dylan Bartusiak \\ Department of Electrical, Computer, and Energy Engineering, University of Colorado, Boulder, Colorado, 80309-0425, USA
}

(Received 18 August 2020; revised 24 January 2021; accepted 8 April 2021; published 20 April 2021)

\begin{abstract}
The differential conductance of metal-insulator-metal devices increases when they are joined with Casimir cavities. An imbalance in injection of hot charge carriers from each side of the insulator is increased with thinner cavities that suppress more quantum vacuum modes. The result is an observed increase in conductance. Additional conductance changes, with insulator thickness and other device parameters, are consistent with an imbalance-induced injection of hot carriers. In addition to the conductance changes, we observe anomalous offsets in the current and voltage. We interpret the conductance changes in terms of a $\Delta E \Delta t$ uncertaintyprinciple-like limit to the injection of hot carriers from zero-point fluctuations.
\end{abstract}

DOI: 10.1103/PhysRevResearch.3.L022007

The effects of zero-point fluctuations (ZPFs) of the electromagnetic field are pervasive. They produce measurable effects through the Casimir force [1,2], Casimir-Polder forces [3-5], radiative shifts such as the Lamb shift [6,7], cavity quantum electrodynamics [8], the Fulling-Davies-Unruh effect [9-11], and the dynamic Casimir effect [12-14]. Because the effects of ZPF are widespread, there is significant interest in using cavities to modify quantum forces and control the interactions between materials and the vacuum field, with applications in many areas including atomic, chemical, microelectronic, biological, and optical systems [4,15-17]. The effects of ZPF can be understood in terms of the strength of the coupling between the field and the matter, which may be atoms, molecules, nanoparticles, semiconductors, and colloidal or biological particles. In strong coupling, there are (i) changes in the energy levels, e.g., Rabi splitting and the formation of polaritons $[18,19]$, (ii) very rapid photon exchange [20], and (iii) often a resonant exchange between the material excitations and the cavity radiation modes [19,21-23]. In weak coupling, (i) the energy level of the materials remains essentially unchanged, and (ii) radiation may be absorbed and emitted, forces modulated, and chemical processes affected $[16,17]$. Here, we describe a possible consequence of weak coupling, a shift in electrical conductance in certain electronic devices, which appears to be an effect of hot carrier generation by ZPFs.

Metal-insulator-metal (MIM) diodes have been investigated to provide ultrahigh frequency rectification for half a century [24]. We make use of internal photoemission, also known as photoinjection, in MIM structures. Photoinjection occurs when electromagnetic radiation incident on an absorber excites hot charge carriers having sufficient energy to surmount an adjacent barrier and enter the interface region

\footnotetext{
*moddel@ colorado.edu

Published by the American Physical Society under the terms of the Creative Commons Attribution 4.0 International license. Further distribution of this work must maintain attribution to the author(s) and the published article's title, journal citation, and DOI.
}

or tunnel through it [25]. Our basic MIM device consists of a thin metal upper electrode, a thin insulator, and a thicker metal lower electrode. Illumination of the upper electrode metal produces hot carriers, and if the electrode is thinner than the hot carrier mean-free path length, which is typically on the order of a few tens of nanometers [26], then the carriers can traverse the metal layer without being scattered. They enter the insulator, which forms the barrier, and traverse it ballistically if it is thinner than the insulator mean-free path length, on the order of several nanometers [27], or tunnel through it if it is thinner still, $<\sim 4 \mathrm{~nm}[28,29]$. After entering the metal base electrode on the other side, the carriers are absorbed [29]. The photoinjected charge influences the electronic properties of MIM devices. In particular, the differential conductance $G$ of the MIM device determined from the slope of the currentvoltage characteristic is increased by the photoinjection of charge [29-31].

In our MIM structures, carriers transit across the combination of the upper electrode and the thin insulator, and their capture in the base electrode can be very fast. In the metal layer, the hot carrier velocity is at least the Fermi velocity of $10^{6} \mathrm{~m} / \mathrm{s}$ [25,32], resulting in a transit time of $<10 \mathrm{fs}$ for a thickness of $\sim 10 \mathrm{~nm}$. In the thinner insulator, ballistic carriers travel at $10^{6} \mathrm{~m} / \mathrm{s}$, resulting in a transit time of $\sim 1$ fs [33], which is like the tunneling time [34] when that is the method of transport. The hot carriers scatter inelastically in the thicker base electrode layer with a lifetime on the order of $\leqslant 10 \mathrm{fs}$ [35]. Thus, the entire process of hot carrier transport and scattering can occur on the order of $10 \mathrm{fs}$.

A Casimir cavity is formed by two closely spaced mirrors separated by a transparent region. The mirrors limit the allowed vacuum electromagnetic modes associated with ZPFs within the cavity. The resulting mode density is described by an Airy function that depends on the reflectivity and spacing of the mirrors [36]. The cavity acts to suppress mostly long wavelengths greater than twice the cavity spacing. This reduces the vacuum energy density inside the cavity below its free-space value [7].

To make the devices described in this paper, we form a Casimir cavity on top of an MIM structure, as shown in Fig. 1. 


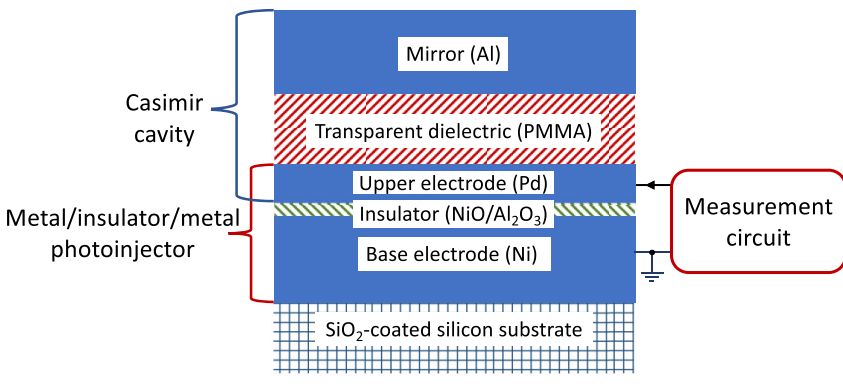

FIG. 1. Cross-section of a Casimir-cavity photoinjector. Except where noted, devices incorporate the materials shown in the figure.

We use these Casimir-cavity photoinjectors to test the effects of ZPFs on the differential conductance of the devices.

In this rapid communication, after describing the device fabrication, we present a series of graphs showing variations in the conductance as a function of device parameters. We describe how the Casimir cavity could break a balance between the injection of charge from opposing electrodes to produce the conductance change. The results are interpreted in terms of the femtosecond response of the device and an uncertaintyprinciple-like relation that has been proposed to constrain interaction with the ZPFs. We also describe an observed anomalous current and our attempts thus far to eliminate it. The observed variations in conductance with the thicknesses of three different parts of the device and with the area provide support for the effects being due to injected carriers excited by ZPFs.

We fabricated Casimir-cavity photoinjector devices using microfabrication techniques that we developed to form MIM diodes for ultrafast rectification applications $[37,38]$. Two different fabrication techniques were employed, with both types of devices showing the variation of conductance described here. Submicron devices were fabricated using a germanium shadow-mask (GSM) process, and larger area devices were fabricated using standard photolithography.

To form devices using the GSM process [37], a 250-nmwide germanium bridge was formed over an $\mathrm{SiO}_{2}$-coated surface of a silicon wafer. The $38-\mathrm{nm}$ Ni base electrode was angle evaporated under the bridge from one side, followed by a combination of native oxide growth of $\mathrm{NiO}$ and sputter deposition of $\mathrm{Al}_{2} \mathrm{O}_{3}$ to form the insulator. We then angle evaporated the Pd upper electrode layer from the opposite side of the bridge. The resulting overlap of the two metals formed an ellipse with an area of $0.02 \pm 0.006 \mu \mathrm{m}^{2}$, as shown in Fig. 2. After we removed the germanium bridge, a Casimir cavity was formed over the MIM structure by spinning on polymethyl methacrylate (PMMA) and coating it with a $150-\mathrm{nm}$ aluminum reflector. In addition to providing a reduced density of ZPF modes, the Casimir cavity encapsulates and stabilizes the MIM structure, blocking further oxidation.

Devices having larger areas were fabricated using standard photolithographic techniques with a $50-\mathrm{nm}$-thick Ni base electrode and a cavity material consisting of sputter-deposited $\mathrm{SiO}_{2}$. Details of device fabrication by both methods are provided in the Supplemental Material [39].

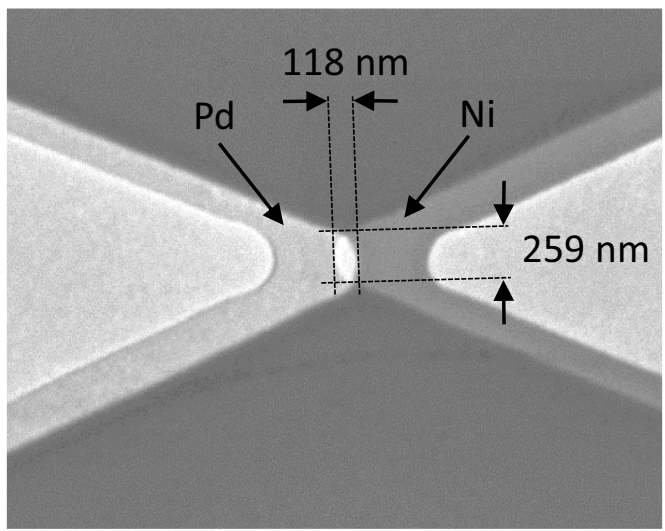

FIG. 2. Scanning electron microscope (SEM) image of a germanium shadow-mask (GSM) metal-insulator-metal (MIM) structure. Arrows indicate the thin Pd upper electrode, the thicker Ni base electrode, and the overlap region in the center, with an area of $0.02 \pm 0.006 \mu \mathrm{m}^{2}$. The remaining lightest regions, in the center and at the far left- and right-hand sides, consist of both $\mathrm{Ni}$ and Pd layers with the insulator layer between them.

We carried out current-voltage measurements using highprecision instrumentation connected in a four-point measurement configuration to avoid the effect of lead resistance. The base electrode is the electrical ground, as shown in Fig. 1, with a positive current corresponding to the direction of the arrow. All measurements were carried out at room temperature. Additional detail is given in the Supplemental Material [39]. We confirmed the reported trends in over 550 MIM-based devices produced in 15 different batches. Virtually all the devices with working (nonshorted) MIM structures exhibited the type of characteristics shown in Fig. 3(a). There were, however, significant device-to-device variations even for devices fabricated under nominally the same conditions. In fabricating and testing tens of thousands of MIM devices, we generally find a wide range of resistances for nominally the same fabrication conditions due to slight variations in the insulator thickness [38], which is $<10$ lattice constants thick. Measurement results presented in Fig. 4 for the standard photolithography devices are averages across each wafer.

The electrical responses of two MIM devices, each with an adjoining Casimir cavity, are shown in Fig. 3(a). The devices for Figs. 3(a) and 3(b) were fabricated using the GSM process. For a device with a 33-nm cavity thickness and a refractive index of approximately 1.5 , the cavity cutoff wavelength approaches $100 \mathrm{~nm}$. Because the absorbance of the PMMA that fills the cavity rises sharply for wavelengths $<250 \mathrm{~nm}$, the actual cutoff wavelength is $>100 \mathrm{~nm}$ [40]. To check that observed variation is not the result of some special property of PMMA, we also measured the thickness dependence for an $\mathrm{SiO}_{2}$-filled cavity and found a similar variation with thickness, as shown in Fig. 3(b). The larger $G$ for the $\mathrm{SiO}_{2}$-filled cavities is likely due to a slightly thinner insulator, and the larger near-ultraviolet (UV) transparency of $\mathrm{SiO}_{2}$ than PMMA [40] (see Table 1 of the Supplemental Material [39]). To check that the $G$ variations are due to the cavity and not just the additional cavity dielectric, we formed devices with and without top mirrors and found that the mirrors are required to change 

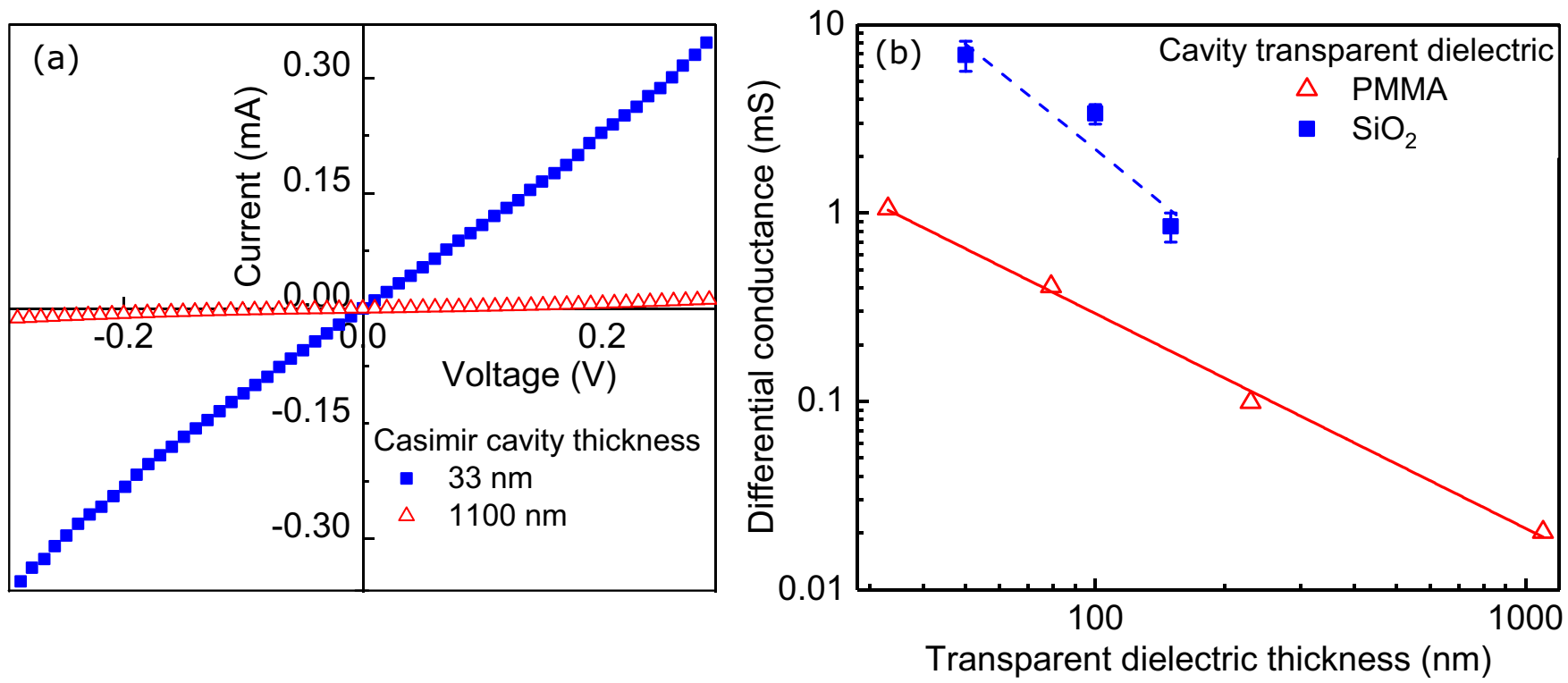

FIG. 3. Effect of Casimir-cavity dielectric thickness. (a) Current as a function of voltage for Casimir photoinjectors having a thin and a very thick polymethyl methacrylate (PMMA) cavity, showing respective differential conductance values at $0 \mathrm{~V}$ and $G$ of $1 \mathrm{mS}$ and $20 \mu \mathrm{S}$. (b) $G$ as a function of cavity thickness. These devices were fabricated using the germanium shadow-mask (GSM) process, with dimensions shown in Fig. 2. The thickness of the Ni base electrode, the insulator effective thickness, and Pd upper electrode thickness are 38, 2.3, and 8.3 nm, respectively, for the PMMA cavity devices, but a thinner 1.9-nm insulator for the $\mathrm{SiO}_{2}$ cavity devices.

$G$, as shown in the Supplemental Material [39]. To check if the conductance changes might somehow result from current leakage through the cavity transparent dielectric, we measured the resistance through the dielectric for three devices with dielectric layer thicknesses ranging from 33 to $79 \mathrm{~nm}$. In each case, the resistance through the transparent dielectric is at least a factor of $10^{6}$ greater than the resistance through the MIM structure, precluding such current leakage as the source for the observed effects.

For an MIM device in equilibrium, any carriers excited by ZPFs and injected into the insulator from the upper electrode would be balanced by ZPF-excited hot carriers from the base electrode. We hypothesize that the presence of the Casimir cavity breaks this balance, resulting in a change in the net injection rate and hence increased $G$. This imbalance is discussed in greater detail after the presentation of the results. Because cavities suppress zero-point electromagnetic modes having wavelengths greater than twice the cavity thickness, reducing the cavity thickness increases the range of suppressed wavelengths. We would therefore expect an increase in $G$ for decreasing cavity thickness, a trend that can be seen in Fig. 3(b).

Examining the results of Fig. 3(b) further, we would expect wavelengths in the visible range to dominate the response because, for near-UV wavelengths $<250 \mathrm{~nm}$, the PMMA becomes highly absorptive [40], and the infrared response is limited because the available vacuum energy density falls off with the cube of the wavelength. Although the vacuum energy density in ideal Casimir cavities varies inversely with the thickness cubed [7], we would not expect the conductance to exhibit such a cubic dependence because there are multiple energy-dependent mechanisms in play, including (i) variations of photoinjection yield with photon energy, as described by extensions of Fowler's theory of photoemission [41]; (ii) the interband transition threshold of Pd [42], which limits the transport of high-energy carriers; (iii) the energy-dependent reflectivity of the mirrors and absorptivity of the transparent dielectric; and (iv) the energy dependence of hot carrier scattering [43].

Additional evidence that the observed effects are due to injected charge can be seen in variations of $G$ with upper electrode and insulator thicknesses. Whatever we can do to increase the photoinjection efficiency will allow a greater suppression of that injection by the Casimir cavity. That, in turn, increases the imbalance between the injection from the base electrode and from the upper electrode and provides a greater net photoinjection rate. As the thickness of the upper electrode increases, a decreasing fraction of photoexcited electrons passes through the metal layer and into the insulator before being scattered, resulting in a falloff of photoinjection efficiency with thickness [44]. Opposing this trend, as the thickness decreases, the incident photon absorption decreases, and the reflectivity decreases, which reduces suppression of electromagnetic modes in the Casimir cavity [45]. The optical absorption depth of $\mathrm{Pd}$ is $\sim 10 \mathrm{~nm}$ for $0.4 \mu \mathrm{m}$ radiation [46]. Increasing the Pd thickness beyond that thickness increases the scattering loss for electrons passing through the upper electrode without substantially increasing the incident photon absorption, and decreasing the thickness reduces the absorption. Therefore, we would expect $G$ to peak roughly at that thickness, which is what we observed, as shown in Fig. 4(a). In general, tunneling conductance decreases exponentially with insulator thickness [47], and therefore, $G$ would follow an exponential trend if its magnitude is determined by charge injection. This is confirmed in Fig. 4(b).

The conductance should vary linearly with device area. This is confirmed for our devices, as shown Fig. 4(c), where $G$ is plotted as a function of device area. The linear variation is 


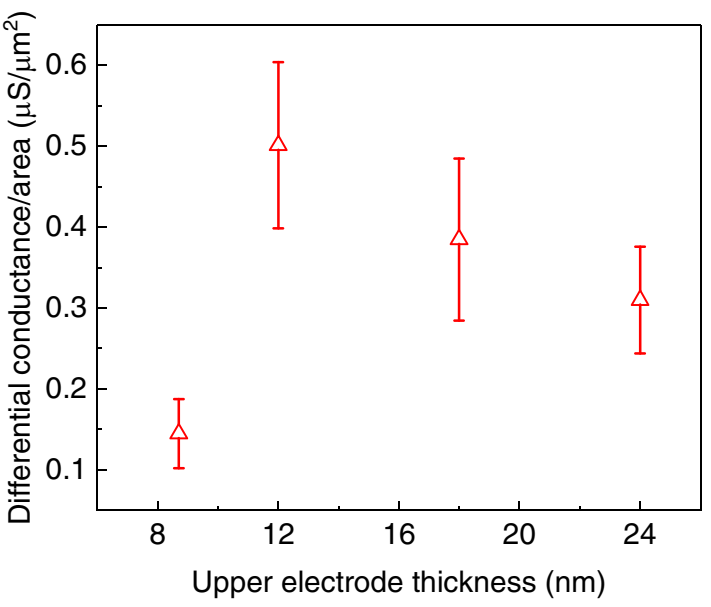

(a)

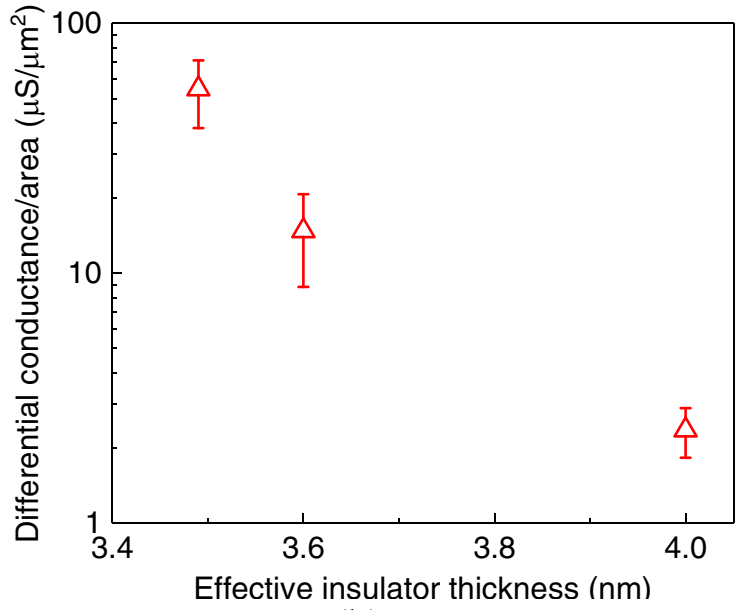

(b)

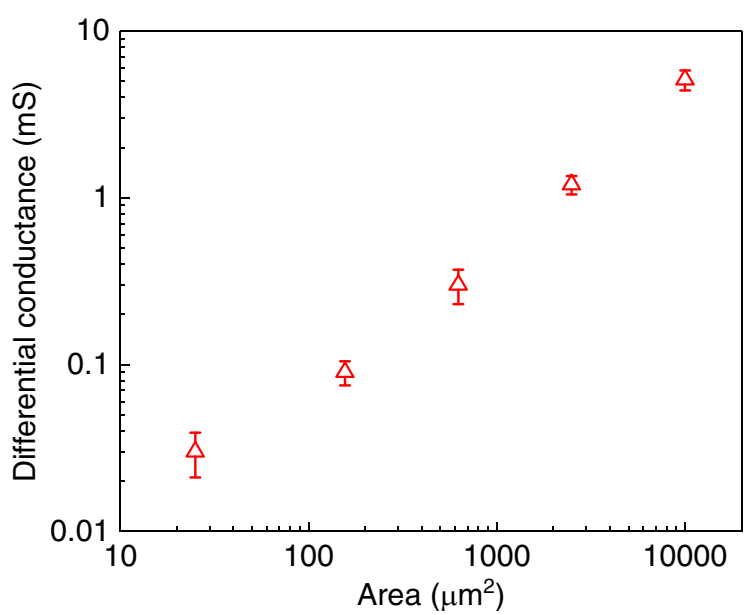

(c)

FIG. 4. Effect of varying charge injection. Differential conductance $(G)$ per unit area as a function of (a) upper electrode thickness and (b) effective insulator thickness. (c) Device area. The insulator effective thickness for (a) and (c) is $5 \mathrm{~nm}$, the Pd upper electrode thickness for (b) and (c) is $12 \mathrm{~nm}$, and the Casimir-cavity $\mathrm{SiO}_{2}$ thickness is $12 \mathrm{~nm}$ for all three sets. The data for (a) and (b) are averaged from devices having areas of 6.25 to $10000 \mu \mathrm{m}^{2}$.

consistent with $G$ being due to the hot electron injection and not an artifact arising from a different part of the device or measurement circuit.

The hot carriers could be electrons, holes, or a combination of the two. For the materials used in the study-Pd for the upper electrode, $\mathrm{NiO}$ and $\mathrm{Al}_{2} \mathrm{O}_{3}$ for the insulator-the effective barrier heights for electrons are $\sim 0.2$ and $0.3 \mathrm{eV}$ for $\mathrm{NiO}$ and $\mathrm{Al}_{2} \mathrm{O}_{3}$, respectively, whereas the respective barrier heights for holes are 3.2 and $5.9 \mathrm{eV}[39,48]$. For that reason, electrons are expected to dominate, but given that the likely transport mechanism is tunneling, probably both electrons and holes are injected [30]. The combination of the two could, in principle, give rise to an increase in conductance with no net current.

In addition to the changes in resistance reported here, a small anomalous offset current and voltage were observed in the Casimir photoinjector devices. This can be seen in Fig. 5, which shows the low voltage range of the devices presented in Fig. 3(a). With the base electrode as ground, a positive current flows from the upper electrode to the base electrode

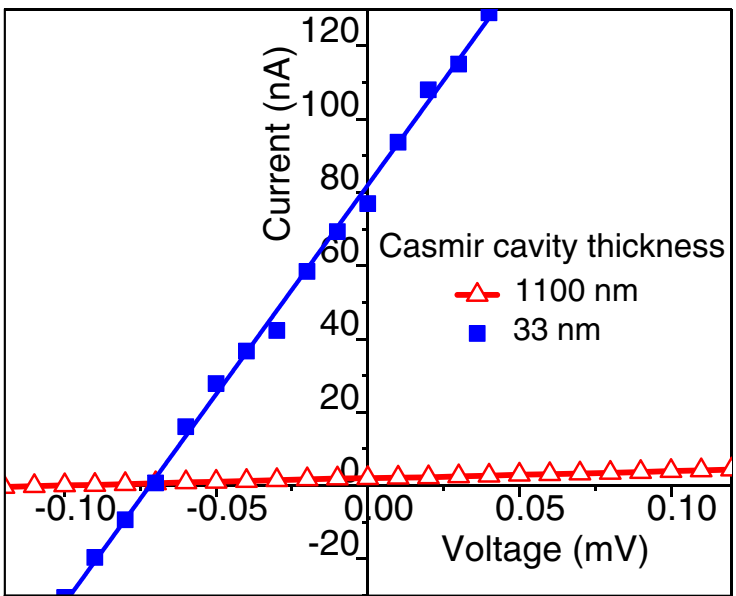

FIG. 5. Small-voltage region of the current-voltage characteristic shown in Fig. 3(a). Because of an anomalous offset, the characteristics do not pass through the origin. 
at $0 \mathrm{~V}$. If we assume that ZPFs cannot be the power source, an external voltage source would be needed. As described in the Supplemental Material [39], we carried out extensive experiments aimed at eliminating this offset, including developing an improved method for measuring low-current electrical characteristics, recalibrating instrumentation, producing temperature gradients to check for thermoelectric effects, making arrays to check that the voltage and current offsets scaled with the respective number of devices in series and in parallel (they did), investigating whether the voltage and current offsets might be transient and possibly a result of hysteresis during measurement (the currents are stable over a period of hours with no externally supplied power), and making sure that the devices are blocked completely from any stray light during measurement. Thus far, we have been unsuccessful in finding an artifact that would explain the anomaly. These offsets are small compared with the voltage and current ranges used to measure the differential conductance values and therefore do not affect those measurements. We describe these currents and voltages in more detail in a separate publication [49].

Further considering the suggested current balance model, virtual hot carriers are excited in the upper electrode metal in response to internal ZPFs in the metal and in response to ZPFs associated with the impinging electromagnetic cavity modes. The hot carrier excitation mechanism may involve plasmonic modes propagating along the Casimir-cavity mirrors [50-52]. The thicker base electrode has no impinging electromagnetic modes to photoexcite hot carriers but compensates for that with a thicker region to provide more hot carriers from internal ZPFs. In equilibrium, the resulting virtual hot carrier injection in one direction balances that in the other direction. The addition of the Casimir cavity reduces the electromagnetic vacuum energy density impinging on the upper electrode to below its magnitude in free space. Our results provide evidence that the cavity upsets this balance and produces injection of real hot carriers across the insulator.

A different but instructive example of the generation of real particles by quantum vacuum fluctuations is the FullingDavies-Unruh effect, where quantum vacuum particles appear virtual to inertial observers but real to accelerated observers
[53]. Another example is the dynamic Casimir effect, where vacuum-state excitations become real photons in response to moving mirrors [12,13]. In our charge-injection devices, we conjecture that it is the presence of the asymmetrically positioned Casimir cavity that manifests the real hot carriers.

We speculate on what special characteristics of ultrahighspeed MIM devices give rise to the observed conductance changes when they are joined with Casimir cavities. As discussed above, the transport and capture time for hot carriers in the MIM devices is several femtoseconds. It has been argued that an amount of energy $\Delta E$ may be borrowed from ZPFs for a time $\Delta t$ following an uncertainty-principle-like relation $[54,55]$, but experiments will likely expand understanding of the constraints involved [56]. For $\Delta E \Delta t \sim \hbar / 2$, hot electrons from $1 \mathrm{eV}$ excitation would be available for $0.3 \mathrm{fs}$. In that case, without sufficiently short transport and capture times, hot carriers would not manifest measurable effects. If this $\Delta E \Delta t$ limitation applies, it would explain the need for ultrafast devices like MIM diodes to produce the observed effects.

In conclusion, the cause for the conductance changes we observe in joining MIM devices with Casimir cavities appears to be the injection of hot carriers into the devices. The changes are consistent with reduced electromagnetic radiation from ZPFs impinging on the device from the cavity side of the device, with larger changes occurring for thinner cavities. Variations in the effect with the photoinjecting metal thickness and transport insulator thickness support the photoinjection model of operation. The reason that such conductance changes occur in MIM devices could be that, for the observed effects to manifest, the ultrafast hot carrier transport and capture of the MIM device is required.

We thank P. C. W. Davies, E. A. Cornell, J. Maclay, and M. J. Estes for insightful comments on our results and M. McConnell and R. Cantwell for help in verifying and improving the measurements. Much of the work was supported by The Denver Foundation, which we gratefully acknowledge. A portion of this work was performed in the UCSB Nanofabrication Facility, an open access laboratory.
[1] H. B. G. Casimir, On the attraction between two perfectly conducting plates, in Proceedings of the Koninklijke Nederlandse Akademie van Wetenschappen (1948), Vol. 51, p. 793.

[2] S. K. Lamoreaux, Demonstration of the Casimir Force in the 0.6 to $6 \mu \mathrm{m}$ Range, Phys. Rev. Lett. 78, 5 (1997).

[3] H. B. G. Casimir and D. Polder, The influence of retardation on the London-van der Waals forces, Phys. Rev. 73, 360 (1948).

[4] M. Bordag, G. L. Klimchitskaya, U. Mohideen, and V. M. Mostepanenko, Advances in the Casimir effect (Oxford University Press, Oxford, 2009), Vol. 145.

[5] D. Dalvit, P. Milonni, D. Roberts, and F. DeRosa (eds.), Casimir Physics, Lecture Notes in Physics (Springer, Berlin, 2011), Vol. 834.

[6] W. E. Lamb Jr. and R. C. Retherford, Fine structure of the hydrogen atom by a microwave method, Phys. Rev. 72, 241 (1947).
[7] P. W. Milonni, The Quantum Vacuum (Academic Press, Boston, 1994).

[8] P. R. Berman (ed.), Cavity Quantum Electrodynamics (Academic Press, San Diego, 1994).

[9] S. A. Fulling, Nonuniqueness of canonical field quantization in Riemannian space-time, Phys. Rev. D 7, 2850 (1973).

[10] P. C. Davies, Scalar production in Schwarzschild and Rindler metrics, J. Phys. A Math. Gen. 8, 609 (1975).

[11] W. G. Unruh, Notes on black-hole evaporation, Phys. Rev. D 14, 870 (1976).

[12] G. Moore, Quantum theory of the electromagnetic field in a variable-length one-dimensional cavity, J. Math. Phys. 11, 2679 (1970).

[13] S. A. Fulling and P. C. Davies, Radiation from a moving mirror in two dimensional space-time: Conformal anomaly, Proc. R. Soc. A 348, 393 (1976). 
[14] C. M. Wilson, G. Johansson, A. Pourkabirian, M. Simoen, J. R. Johansson, T. Duty, and P. Delsing, Observation of the dynamical Casimir effect in a superconducting circuit, Nature 479, 376 (2011).

[15] R. Miller, T. E. Northup, K. M. Birnbaum, A. Boca, A. D. Boozer, and H. J. Kimble, Trapped atoms in cavity QED: Coupling quantized light and matter, J. Phys. B 38, S551 (2005).

[16] D. P. Sheehan, Casimir chemistry, J. Chem. Phys. 131, 104706 (2009).

[17] J. Galego, C. Climent, F. J. Garcia-Vidal, and J. Feist, Cavity Casimir-Polder Forces and Their Effects in Ground-State Chemical Reactivity, Phys. Rev. X 9, 021057 (2019).

[18] R. F. Ribeiro, L. A. Martínez-Martínez, M. Du, J. CamposGonzalez-Angulo, and J. Yuen-Zhou, Polariton chemistry: Controlling molecular dynamics with optical cavities, Chem. Sci. 9, 6325 (2018).

[19] E. Orgiu, J. George, J. A. Hutchison, E. Devaux, J. F. Dayen, B. Doudin, F. Stellacci, C. Genet, J. Schachenmayer, C. Genes, G. Pupillo, P. Samorì, and T. W. Ebbesen, Conductivity in organic semiconductors hybridized with the vacuum field, Nat. Mater. 14, 1123 (2015).

[20] A. Canaguier-Durand, E. Devaux, J. George, Y. Pang, J. A. Hutchison, T. Schwartz, C. Genet, N. Wilhelms, J. M. Lehn, and T. W. Ebbesen, Thermodynamics of molecules strongly coupled to the vacuum field, Angewandte Chemie Int. Ed. 52, 10533 (2013).

[21] G. Khitrovai, H. M. Gibbs, M. Kira, S. W. Koch, and A. Scherer, Vacuum Rabi splitting in semiconductors, Nat. Phys. 2, 81 (2006).

[22] J. Feist and F. J. Garcia-Vidal, Extraordinary Exciton Conductance Induced by Strong Coupling, Phys. Rev. Lett. 114, 196402 (2015).

[23] J. Galego, F. J. Garcia-Vidal, and J. Feist, Suppressing photochemical reactions with quantized light fields, Nat. Commun. 7, 13841 (2016).

[24] M. Heiblum, S. Wang, J. Whinnery, and T. Gustafson, Characteristics of integrated MOM junctions at DC and at optical frequencies, IEEE J. Quantum Electron. 14, 159 (1978).

[25] R. Williams, Injection by internal photoemission, in Semiconductors and Semimetals (Academic Press, New York, 1970), Vol. 6, pp. 97-139.

[26] D. Gall, Electron mean free path in elemental metals, J. Appl. Phys. 119, 085101 (2016).

[27] L. Nelson and D. E. Anderson, Hot-electron transfer through thin-film Al- $\mathrm{Al}_{2} \mathrm{O}_{3}$ triodes, J. Appl. Phys. 37, 66 (1966).

[28] J. G. Simmons, Potential barriers and emission-limited current flow between closely spaced parallel metal electrodes, J. Appl. Phys. 35, 2472 (1964).

[29] J. Kadlec and K. H. Gundlach, Results and problems of internal photoemission in sandwich structures, Phys. Status Solidi A 37, 11 (1976).

[30] D. A. Kovacs, J. Winter, S. Meyer, A. Wucher, and D. Diesing, Photo and particle induced transport of excited carriers in thin film tunnel junctions, Phys. Rev. B 76, 235408 (2007).

[31] F. Wang and N. A. Melosh, Theoretical analysis of hot electron collection in metal-insulator-metal devices, in Next Generation (Nano) Photonic and Cell Technologies for Solar Energy
Conversion II (International Society for Optics and Photonics, Bellingham, WA, 2011), Vol. 8111, p. 811100.

[32] E. Knoesel, A. Hotzel, and M. Wolf, Ultrafast dynamics of hot electrons and holes in copper: Excitation, energy relaxation, and transport effects, Phys. Rev. B 57, 12812 (1998).

[33] M. Heiblum and M. V. Fischetti, Ballistic hot-electron transistors, IBM J. Res. Dev. 34, 530 (1990).

[34] H. Q. Nguyen, P. H. Cutler, T. E. Feuchtwang, Z.-H. Huang, Y. Kuk, P. J. Silverman, A. A. Lucas, and T. E. Sullivan, Mechanisms of current rectification in an STM tunnel junction and the measurement of an operational tunneling time, IEEE Trans. Electron Devices 36, 2671 (1989).

[35] M. Bauer, A. Marienfeld, and M. Aeschlimann, Hot electron lifetimes in metals probed by time-resolved two-photon photoemission, Prog. Surf. Sci. 90, 319 (2015).

[36] A. Lambrecht, M. T. Jaekel, and S. Reynaud, The Casimir force for passive mirrors, Phys. Lett. A 225, 188 (1997).

[37] B. Pelz and G. Moddel, Demonstration of distributed capacitance compensation in a metal-insulator-metal infrared rectenna incorporating a traveling-wave diode, J. Appl. Phys. 125, 234502 (2019); see supplement for additional fabrication information.

[38] S. B. Herner, A. Belkadi, A. Weerakkody, B. Pelz, and G. Moddel, Responsivity-resistance relationship in MIIM diodes, IEEE J. Photovolt. 8, 499 (2018).

[39] See Supplemental Material at https://link.aps.org/supplemental/ 10.1103/PhysRevResearch.3.L022007 for details about device fabrication and measurement.

[40] H. M. Zidan and M. Abu-Elnader, Structural and optical properties of pure PMMA and metal chloride-doped PMMA films, Physica B 355, 308 (2005).

[41] H. Chalabi, D. Schoen, and M. L. Brongersma, Hot-electron photodetection with a plasmonic nanostripe antenna, Nano Lett. 14, 1374 (2014).

[42] G. Tagliabue, A. S. Jermyn, R. Sundararaman, A. J. Welch, J. S. DuChene, R. Pala, A. R. Davoyan, P. Narang, and H. A. Atwater, Quantifying the role of surface plasmon excitation and hot carrier transport in plasmonic devices, Nat. Commun. 9, 3394 (2018).

[43] R. Ludeke, A. Bauer, and E. Cartier, Hot electron transport through metal-oxide-semiconductor structures studied by ballistic electron emission spectroscopy, J. Vac. Sci. Technol. B Microelectron. Nanometer Struct. Process. Meas. Phenom. 13, 1830 (1995).

[44] V. L. Dalal, Simple model for internal photoemission, J. Appl. Phys. 42, 2274 (1971).

[45] M. Lisanti, D. Iannuzzi, and F. Capasso, Observation of the skin-depth effect on the Casimir force between metallic surfaces, Proc. Natl. Acad. Sci. 102, 11989 (2005).

[46] A. Borghesi, G. Guizzetti, and Palladium, Handbook of Optical Constants of Solids II, edited by E. D. Palik (Academic, New York, 1991), p. 469.

[47] J. G. Simmons, Generalized formula for the electric tunnel effect between similar electrodes separated by a thin insulating film, J. Appl. Phys. 34, 1793 (1963).

[48] A. Weerakkody, A. Belkadi, and G. Moddel, Nonstoichiometric nanolayered $\mathrm{Ni} / \mathrm{NiO} / \mathrm{Al}_{2} \mathrm{O}_{3} / \mathrm{CrAu}$ metal-insulatormetal infrared rectenna, ACS Appl. Nano Mater. 4, 2470 (2021). 
[49] G. Moddel, A. Weerakkody, D. Doroski, and D. Bartusiak, Optical-cavity-induced current, Symmetry 13, 517 (2021).

[50] M. Bordag, The Casimir effect for thin plasma sheets and the role of the surface plasmons, J. Phys. A Math. Gen. 39, 6173 (2006).

[51] F. Intravaia, C. Henkel, and A. Lambrecht, Role of surface plasmons in the Casimir effect, Phys. Rev. A 76, 033820 (2007).

[52] H. Iizuka and S. Fan, Casimir force between two plasmonic metallic plates from a real frequency perspective, J. Opt. Soc. Am. B 36, 2981 (2019).
[53] G. E. Matsas and D. A. Vanzella, The Fulling-Davies-Unruh effect is mandatory: The proton's testimony, Int. J. Mod. Phys. D 11, 1573 (2002).

[54] L. H. Ford, Constraints on negative-energy fluxes, Phys. Rev. D 43, 3972 (1991).

[55] P. C. W. Davies and A. C. Ottewill, Detection of negative energy: 4-dimensional examples, Phys. Rev. D 65, 104014 (2002).

[56] J. Maclay and E. W. Davis, Testing a quantum inequality with a meta-analysis of data for squeezed light, Found. Phys. 49, 797 (2019). 